Review Article

\title{
N-Acetylcysteine: A Review of Clinical Usefulness (an Old Drug with New Tricks)
}

\author{
Gerry K. Schwalfenberg (iD \\ Department of Family Medicine, University of Alberta, No. 301, 9509-156 Street, Edmonton T5P 4J5, AB, Canada \\ Correspondence should be addressed to Gerry K. Schwalfenberg; gschwalf@shaw.ca
}

Received 25 March 2021; Accepted 26 May 2021; Published 10 June 2021

Academic Editor: Tatiana Emanuelli

Copyright (C) 2021 Gerry K. Schwalfenberg. This is an open access article distributed under the Creative Commons Attribution License, which permits unrestricted use, distribution, and reproduction in any medium, provided the original work is properly cited.

\begin{abstract}
Objective. To review the clinical usefulness of $\mathrm{N}$-acetylcysteine (NAC) as treatment or adjunctive therapy in a number of medical conditions. Use in Tylenol overdose, cystic fibrosis, and chronic obstructive lung disease has been well documented, but there is emerging evidence many other conditions would benefit from this safe, simple, and inexpensive intervention. Quality of Evidence. PubMed, several books, and conference proceedings were searched for articles on NAC and health conditions listed above reviewing supportive evidence. This study uses a traditional integrated review format, and clinically relevant information is assessed using the American Family Physician Evidence-Based Medicine Toolkit. A table summarizing the potential mechanisms of action for $\mathrm{N}$-acetylcysteine in these conditions is presented. Main Message. N-acetylcysteine may be useful as an adjuvant in treating various medical conditions, especially chronic diseases. These conditions include polycystic ovary disease, male infertility, sleep apnea, acquired immune deficiency syndrome, influenza, parkinsonism, multiple sclerosis, peripheral neuropathy, stroke outcomes, diabetic neuropathy, Crohn's disease, ulcerative colitis, schizophrenia, bipolar illness, and obsessive compulsive disorder; it can also be useful as a chelator for heavy metals and nanoparticles. There are also a number of other conditions that may show benefit; however, the evidence is not as robust. Conclusion. The use of $\mathrm{N}$-acetylcysteine should be considered in a number of conditions as our population ages and levels of glutathione drop. Supplementation may contribute to reducing morbidity and mortality in some chronic conditions as outlined in the article.
\end{abstract}

\section{Introduction}

$\mathrm{N}$-acetylcysteine (NAC) is a sulfhydryl-containing compound, with mucolytic properties, originally patented in 1960, and its use in medicine was first reported in 1967 [1]. Its chemical structure and nomenclature are depicted in Figure 1. Clinically it has been used in cystic fibrosis since 1969 [2]. Since then, NAC use has been expanded to acetaminophen overdose and chronic obstructive lung disease and its role is ever expanding clinically.

Cysteine is found naturally in meat, fish, grains, dairy, soybean, and egg products [3]. As a nutritional supplement, NAC is found in small amounts naturally in some fruits and vegetables [4].

The properties of NAC include enhancing glutathione S-transferase activity, repleting glutathione, scavenging free radicals, and stabilizing protein structures by crosslinking cysteine disulfide molecules along with its antioxidant, antiinflammatory, and mucolytic properties. A more complete list of mechanisms of action is given in Table 1.

The bioavailability of oral NAC in humans is between 4 and $9.1 \%$ in one study [20] and between 6 and $10 \%$ in another [21]; thus, studies using less than $1200 \mathrm{mg}$ per day may show no significant benefit. The half-life of NAC is 6.25 hours, and clearance is both renal and nonrenal, with side effects of nausea, vomiting, and diarrhea [22].

Some have suggested that cysteine deficiency as we age is responsible for loss of youth and loss of health and quality of life and contributes to sarcopenia, especially since cysteine consumption is considered suboptimal [23].

There have been several systematic reviews of NAC in the literature over the past decade looking at various clinical 


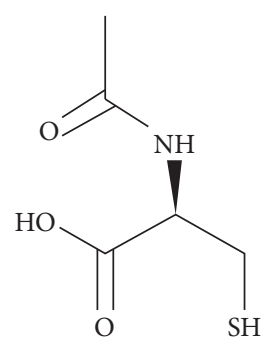

FIgURE 1: N-acetylcysteine formula. $\mathrm{N}$-acetylcysteine may otherwise be called N-acetyl-L-cysteine, NAC, or NALC. Trade names for $\mathrm{N}$-acetylcysteine are Mucomyst, Fluimucil, and Acetadote. The molecular formula is C5H9NO3S. Its chemical structure is shown (source is https://en.wikipedia.org/wiki/Acetylcysteine).

trials in psychiatry and neurology [24], metabolic disease [25], pulmonary disease [26], infectious diseases including possible use in acute respiratory syndromes like corona virus 2 (SARS-CoV-2) [27], and infertility [28] and as chelator for metal toxicity [14].

\section{Methods}

This review of NAC and its relevance in clinical practice was prepared by assessing medical and scientific literature available on MEDLINE and PubMed as well as by reviewing books and conference proceeding. A traditional integrated review format was used in this review where there is limited primary study, thus endeavoring to provide a useful overview of the literature as it pertains to clinical uses for the clinician. The literature reviewed was from the past forty years with emphasis on human studies with preference for randomized control trial (RCT) studies or meta-analysis. Preclinical studies in cell cultures or animal studies were added when relevant. The terms searched for were $\mathrm{N}$-acetylcysteine, NAC, acetaminophen, cystic fibrosis, chronic obstructive lung disease, asthma, bronchitis, bronchiectasis, idiopathic pulmonary fibrosis, sarcopenia, Parkinson's disease, dementia, multiple sclerosis, acquired immune deficiency syndrome (AIDS), tuberculosis, influenza, SARSCoV-2, glutamate, glutathione, contrast nephropathy, schizophrenia, autism, obsessive compulsive disorder, polycystic ovary disease, male infertility, sleep apnea, cancer, hypertension, ulcerative colitis, and others.

The American Family Physician Evidence-Based Medicine Toolkit was used to determine the level of evidence where clinically relevant information was assessed [29]. Assessing level of evidence is important for clinicians and this tool kit was used to provide a sense of the strength of recommendations based on a body of evidence.

An overview of the use of NAC in clinically relevant disorders is depicted in Figure 2.

2.1. N-Acetylcysteine in Lung Disorders. Studies on lung disorders in humans is an expanding topic with early beginnings using inhaled Mucomyst for cystic fibrosis. Oral NAC is now being used for conditions like chronic obstructive lung disease and other conditions as outlined below.
2.2. Cystic Fibrosis $(L O E=A)$. Cystic fibroses $(C F)$ is a multiorgan genetic recessive disorder that affects $1 / 2500$ births and is characterized by hyperviscoelastic sputum, neutrophilic inflammation, and infection.

High-dose oral NAC was used safely in modulating inflammation, improving glutathione levels, and decreasing elastase activity [30]. A recent study showed that lung function was maintained with oral NAC over 24 weeks, while there was deterioration on placebo; however, other markers of inflammation did not change [31]. A 1999 Cochrane Review of nebulized NAC did not show significant benefit and nebulized NAC had a very bad taste and smell. Oral NAC studies were small and only showed minimal benefit [32]. However, a recent study on nebulized NAC did show protection of lung function in children [33]. Distal intestinal obstruction found in CF patients may benefit from a nonsurgical alternative using NAC although further studies are warranted [34].

\subsection{Chronic Obstructive Lung Disease and Chronic Bronchitis} $(L O E=A, C)$. As early as 1985, it was reported that, in chronic obstructive lung disease (COPD), polymorphonuclear leukocyte oxidative damage was reduced with the use of NAC in a controlled in vitro cell model [35]. More recently, a meta-analysis looked at chronic bronchitis and COPD treated with NAC, and there were significantly fewer exacerbations in the treated group than in the placebo group. For those with documented airway obstruction, recommendations are to take $1200 \mathrm{mg} /$ day, as a preventative [36].

A quantitative systematic review using NAC in chronic bronchitis showed significant benefit in preventing exacerbation and the number needed to treat (NNT) to achieve benefit was 5.8 [37]. Recommendations as add-on therapy for COPD and chronic bronchitis is considered a reasonable approach [38].

2.4. Asthma and Allergy $(L O E=B)$. Studies at present do not support the use of NAC in acute asthma attacks because of lack of improvement of cough, wheezing, dyspnea, sputum expectoration, or night sleep [39]. However, in animal models, steroid-resistant acute exacerbation of asthma did benefit from NAC [40]. To date, there have been no longterm studies using NAC in prevention of recurrent asthma attacks by reducing inflammation and mucous plugging. NAC has been shown to reduce the allergen-induced nasal inflammatory cascade in allergic rhinitis in animal models. [41]. Topical application of NAC prior to ragweed exposure resulted in attenuation of the late phase allergic response mediated nasal symptoms [42].

2.5. Bronchiectasis $(L O E=A)$. There is in vitro evidence that biofilms are disrupted and prevented with use of NAC potentially reducing infection [43]. A recent long-term RCT study using NAC $600 \mathrm{mg}$ bid resulted in fewer exacerbations (RR 0.41), less 24-hour mucous production, and a significant improvement in quality of life [44]. 
TABLE 1: N-acetylcysteine (NAC) potential mechanisms of action.

\begin{tabular}{|c|c|c|}
\hline 1 & Action on glutathione & $\begin{array}{l}\text { NAC restores glutathione (cysteine is rate limiting) [5] as seen in cell and animal studies } \\
\text { and clinically in acetaminophen overdose. }\end{array}$ \\
\hline 2 & Stabilizes proteins/DNA & $\begin{array}{l}\text { Protects proteins by crosslinking cysteine disulfide molecules [6]. Various mechanisms } \\
\text { of DNA repair/protection [7] as seen in animal studies and human cell studies. }\end{array}$ \\
\hline 3 & Scavenges free radicals & Scavenging property via the redox potential of thiols [8] as demonstrated in cell culture. \\
\hline 4 & Anti-inflammatory property & Reduces proinflammatory cytokines [9] as seen in animal studies. \\
\hline 5 & Antioxidant property & Reduces oxidative damage [10] as seen in cell cultures. \\
\hline 6 & Mucolytic property & $\begin{array}{l}\text { Splits disulfide bonds in mucoproteins lowering viscosity [11] demonstrated in purified } \\
\text { mucus gels and tracheal explant systems and in vitro (in a pig tracheal pouch) models. }\end{array}$ \\
\hline 7 & Mitochondrial resilience & $\begin{array}{l}\text { Neurogenesis-inducing ability [12] reduces apoptosis of mitochondria [13] as } \\
\text { demonstrated in human dental pulp cells. }\end{array}$ \\
\hline 8 & Metal chelation & Thiol groups provide binding sites for metals [14] in animal studies. \\
\hline 9 & Glutamate/dopamine homeostasis & Modulates glutamate and dopamine [15] extensive studies in humans. \\
\hline 10 & Antiviral properties & $\begin{array}{l}\text { Immune modulation, anti-NF-KB properties, and other unexplored mechanisms [16] } \\
\text { observed in vitro and in vivo. }\end{array}$ \\
\hline 11 & Vascular endothelial growth factor & Inhibition of vascular permeability [17] as seen in human keratinocytes. \\
\hline & $\begin{array}{l}\text { Adenosine triphosphate (ATP) and nitric } \\
\text { oxide (NO) production }\end{array}$ & $\begin{array}{l}\text { Increased ATP production in some cells like fibroblasts in vitro [18]. Increased nitric } \\
\text { oxide production [19] as demonstrated in human studies. }\end{array}$ \\
\hline
\end{tabular}

2.6. Bronchiolitis $(L O E=A)$. Nebulized NAC used in children was found to be effective by reducing the clinical severity score within 3-5 days and resulted in earlier discharge [45]. Further studies are in progress.

2.7. Idiopathic Pulmonary Fibrosis $(L O E=A)$. Idiopathic pulmonary fibrosis is a fatal lung disease with limited options and poor prognosis. In 2011, the use of three medications Azathioprine, Prednisone, and NAC in combination was halted because of increased hospitalizations and deaths. The evidence against the use of these three medications together was strong [46]. Evidence has been mixed over the years but more recently a systematic review and meta-analyses have provided more clarity in the clinical use of NAC in pulmonary fibrosis showing benefit in improving oxygenation and reduced the decline in lung function; however, complications and mortality have remained similar [47].

\section{Liver and Bowel Diseases}

3.1. Acetaminophen Overdose $(L O E=A)$. The usefulness of NAC to prevent acute liver failure has been well established if used according to well-established intravenous dose protocols within 8 hours of ingestion [48]. NAC as an antidote for acetaminophen-induced (AI) liver damage was first written about in 1977 and was more widely accepted by the mid 1980s [49]. The reactive metabolite of acetaminophen is detoxified by glutathione. NAC restores glutathione levels, thus preventing irreversible damage.

3.2. Non-Acetaminophen-Induced Acute Liver Failure $(L O E=A)$. The etiology of non-acetaminophen-induced acute liver failure (NAI-ALF) may include agents like viruses, drugs, toxins, herbal and traditional medications, and autoimmunity. Treatment includes removing the offending agent or specific treatments for the agent. NAC has been used in mushroom poisoning [50], herbicide (Paraquat) poisoning [51], chloroform poisoning [52], and protecting against polychlorinated biphenyls (PCB) induced steatosis [53] and other poisonings. NAC has been used because of its antioxidant, anti-inflammatory, and vasodilating effects as seen in acetaminophen damage [54]. The use of NAC in NAI-ALF reduced mortality and average length of stay and improved survival $[55,56]$. A meta-analysis of prospective clinical trials reviewing NAC-treated and placebo-treated groups showed NAC to be safe and prolonged the survival of patients with native livers without transplantation but did not improve overall survival. [57].

3.3. Hepatocarcinoma $(L O E=B)$. Liver cancer, most commonly known as hepatocarcinoma, is a common malignancy, and treatment of this cancer with interferon-alpha $2 \mathrm{~A}$ $($ IFN- $\alpha$ ) has a relatively poor response rate of about $30 \%$. NAC acts synergistically to improve the efficacy of the drug by decreasing tumor viability, increasing apoptosis, and decreasing expression of nuclear factor kappa-light-chainenhancer of activated B cells (NF-kB). Inactivating the pathway of initiation, promotion, and progression of tumors by NF-kB can be done by using NAC as an adjuvant to IFN $\alpha$ [58].

3.4. Crohn's Disease $(L O E=A)$. Crohn's disease is characterized by marked systemic oxidative stress even in those in clinical remission [59]. In a double-blind RCT $(N=168)$, the relapse rate of those on NAC $400 \mathrm{mg}$ twice daily was significantly reduced compared to placebo while tapering off prednisolone [60].

3.5. Ulcerative Colitis $(L O E=A)$. The use of antioxidant therapy in inflammatory bowel disease has been suggested in the past [61]. In an RCT, using $400 \mathrm{mg}$ of NAC twice a day in ulcerative colitis patients who were on prednisolone taper, the relapse rate was significantly less in the treatment group. 




\footnotetext{
Miscellaneous disorders and conditions that may benefit from NAC:

(i) Systemic lupus erythematosus

(ii) Various types of cancer as well as mitigation of toxicity of some chemotherapy agents

(iii) Chelation of various toxic metals especially lead, chromium, and arsenic

(iv) Mitigation of nanoparticle toxicity on DNA methylation
}

Figure 2: A graphic representation of the clinical uses of $\mathrm{N}$-acetylcysteine (NAC) in various organ systems, as treatment or adjuvant therapy.

The endoscopic relapse rate, serum level of high-sensitivity C-reactive protein (hs-CRP), and fecal calprotectin level were all lower in the treatment group [62].

3.6. Systemic Lupus Erythematosus $(L O E=A)$. There is depletion of glutathione in patients with systemic lupus erythematosus as well as T-cell dysfunction. NAC replenishes glutathione and as an antioxidant in and of itself is able to inhibit mechanistic target of rapamycin (mTOR) in vitro [62]. A double-blind RCT pilot study using $2.4 \mathrm{gm}$ of NAC daily safely and significantly improved lupus disease activity [63].

\section{Metabolic Syndrome including Nonalcoholic Fatty Liver Disease, Diabetes, and Polycystic Ovary}

4.1. Nonalcoholic Fatty Liver Disease $(L O E=B)$. There is evidence that NAC may block hepatic lipid accumulation and provide therapeutic benefit against metabolic 
complications found in nonalcoholic fatty liver disease (NAFLD). This is primarily due to the antioxidant effects and attenuation of lipid peroxidation [64]. This is supported by most preclinical studies and a few clinical studies, and there is an urgent need for larger clinical studies. This disorder affects up to $25 \%$ of the population, a condition which may lead to significant pathology such as fibrosis of the liver.

4.2. Diabetes. Animal studies show that NAC may inhibit hepatic steatosis and development of glucose intolerance and improve lipid profiles $[65,66]$. In type 2 diabetic humans, there does not appear to be any benefit in improving glucose tolerance or $\beta$-cell function with the addition of NAC in short-term trials (2 weeks) [67]. Long-term trials are warranted.

4.3. Diabetic Neuropathy, Retinopathy, and Nephropathy $(L O E=A)$. NAC has been shown to benefit diabetic peripheral neuropathy in animals [68]. Likewise, animal studies investigating retinopathy [69] and diabetic nephropathy [70] show promising results. Recently, a study in humans has shown significant benefit in painful peripheral neuropathy [71]. Patients with painful neuropathy improved over 8 weeks with the use of NAC (600 mg bid) as adjuvant therapy to pregabalin. There was a $>50 \%$ reduction in pain score compared to placebo.

4.4. Polycystic Ovary Disease, Chorioamnionitis, and Recurrent Pregnancy Loss $(L O E=A, B)$. Treatment with NAC may improve insulin sensitivity in women with polycystic ovary disease [72]. A systematic review of RCTs using NAC as a supplement in polycystic ovary disease resulted in improved fertility, ovulation, and odds of having a live birth although these results were not as robust as studies using Metformin [73]. The dosage in studies varied from $1200 \mathrm{mg} /$ day to $1800 \mathrm{mg} /$ day. Chorioamnionitis, a devastating infection with increased risk of cerebral palsy and other neurological sequalae, may benefit from intravenous NAC (both antenatally and postnatally) by reducing neuroinflammation [74]. The use of NAC as an adjunct in recurrent pregnancy loss has been shown to improve the take-home-baby rate as compared to folic acid alone [75].

4.5. Male Fertility $(L O E=A)$. In idiopathic male infertility, an RCT using NAC showed improvement of oxidative status along with semen quality (improved motility, viscosity, and volume) [76]. The dosage was $600 \mathrm{mg}$ twice daily. In another RCT using selenium and NAC together, there was significant improvement in semen quality [77].

4.6. Hypertension $(L O E=B)$. Cysteine-rich diets such as the dietary approaches to stop hypertension (DASH) diet improve insulin resistance, decrease oxidative stress, lower advanced glycation end products, increase the storage form of glutathione, and modulate nitric oxide and other vasoactive molecules, thus lowering blood pressure [3]. However, NAC as add-on therapy in nondiabetic patients with chronic kidney disease who were on renin-angiotensin system blockade medication had no effect on blood pressure [78]. N-acetylcysteine $1800 \mathrm{mg}$ daily over 1 month significantly lowered homocysteine levels and systolic and diastolic blood pressure in middle-aged men who were hyperlipidemic [79]. The combination of NAC and L-arginine in diabetic patients results in increased nitric oxide production and improves systolic blood pressure [19].

4.6.1. Pulmonary Hypertension. There is some evidence that NAC may inhibit the development of pulmonary artery hypertension $(\mathrm{PAH})$ or significantly reduce pulmonary vascular remodeling in animal models [80, 81]. Previous information was contrary to this [82] and it was thought that $\mathrm{PAH}$ was a side effect of NAC.

4.7. Chemotherapy. Cyclophosphamide is known to have cardiotoxicity which may be mitigated by NAC [83] and cyclophosphamide may cause hemorrhagic cystitis which may be prevented with NAC [84]. Acute kidney damage as seen with cisplatin may be reduced as seen in animal studies [85]. In pediatric cancers studied in mouse models, the use of NAC blocks the side effects of cisplatin but may interfere with its effectiveness if used together. The use of NAC needs to be separated from chemotherapy by at least 4 hours [86].

4.8. Breast Cancer, Prostate Cancer, Lung Cancer, Glioblastoma, and Chronic Lymphocytic Leukemia. There are many studies looking at the effect of NAC on cancer cells. By changing the environment in and surrounding the cells, there appears to be benefit in both human and cell studies.

There has been a human pilot study determining that NAC has antiproliferative effects on breast cancer [87]. NAC markedly reduces monocarboxylate transporter 4 (MCT4) transporter proteins from being utilized to import energy as lactate to cancer cells. MCT4 is considered a marker of aggressive cancer behaviour with poor overall survival.

In a prostate cancer cell study, NAC suppressed prostate cancer cell growth [88] and prevented adhesion and invasion to remote locations [88].

Cell studies in lung cancer show that NAC has the ability to detoxify chemicals as a precursor of reduced glutathione, scavenging of radicals, and protection from DNA damage. However, there is some caution since vitamin E and NAC in a combination study showed increased tumor cell proliferation by reducing reactive oxygen species and reducing p53 expression (which increases tumor growth) in mouse and human lung tumor cells [89], whereas NAC and a major tea polyphenol epigallocatechin-3-gallate (EGCG) form an adduct which may enhance EGCG cell killing of cancer cells [90].

Imatinib is an agent successfully used in chronic lymphocytic leukemia and NAC enhances its effectiveness in animal models by increasing the production of nitric oxide [91]. 
In glioblastoma cell studies, NAC has the remarkable ability to inhibit tumor growth and cell proliferation [92]. Further studies regarding NAC and its role in cancer therapy are needed.

4.9. Sleep Apnea $(L O E=B)$. Sleep apnea has become a significant problem that may lead to hypertension, stroke, and various cardiovascular ailments. This condition is considered a proinflammatory vascular risk factor and NAC has been thought to improve this [93]. There are small clinical RCT trials that have shown benefit $[94,95]$.

\section{Infectious Disease}

5.1. Overview. NAC may strengthen immune defence by increasing the glutathione pool in leukocytes, thus decreasing the likelihood of infections [96].

5.2. Acquired Immune Deficiency Syndrome $(L O E=A)$. In a double-blind placebo-controlled trial using $800 \mathrm{mg}$ of NAC, there was a reduction in the decline of the CD4 count (number of a type of white blood cell) seen in the placebo group and tumor necrosis factor-alpha (TNF-alpha) levels were also reduced [97]. Glutathione levels are improved with NAC, inhibiting actions of inflammatory cytokines and slowing cachexia and wasting [98].

5.3. Tuberculosis $(L O E=A)$. Clinically, NAC as add-on therapy for tuberculosis (TB) treatment significantly improved clearing of infiltration and reduction in cavity size radiologically and brought about faster sputum negativity [99]. In hospitalized TB patients, NAC was associated with a significant reduction in all-cause mortality within 90 days of admission [100].

5.4. Influenza, Respiratory Syncytial Virus, and SARS-CoV-2 $(L O E=A, B)$. A double-blind RCT in which 262 patients were given $600 \mathrm{mg}$ of NAC or placebo for 6 months (during the winter) was conducted to determine the effect of longterm treatment on influenza. This study showed that only $25 \%$ of virus-infected patients in the NAC group were symptomatic compared to $79 \%$ of patients in the placebo group. There was a significant decrease in influenza-like episodes, severity, and length of time confined to bed and sharp reduction of both local and systemic symptoms in the NAC group. Replication of seasonal human influenza A viruses is inhibited by NAC along with inhibition of virusinduced proinflammatory responses [101].

In cell cultures, inhibition of mucin synthesis and reduction of proinflammatory mediators are seen in alveolar type II epithelial cells which are infected with respiratory syncytial virus (RSV) and influenza A and B viruses [102].

Thiols block the angiotensin-converting enzyme 2, thereby inhibiting penetration of SARS-CoV-2 into cells. In [103], recent reviews of NAC and SARS-CoV-2 (COVID-19) show promise as an agent to modify the immune response and possibly reduce morbidity and mortality $[27,104]$. In a case report about one patient with severe COVID-19, NAC demonstrated significant benefit [105]. There are several studies that are in progress.

5.5. H. pylori $(L O E=B)$. The additive effect of NAC in the usual treatment of Heliobacter pylori $(H$. pylori) appears to improve eradication rates by reducing mucus [106, 107]. It is suggested that NAC may prevent gastritis induced by the organism [108]. In a Cochrane review, the evidence from most studies is weak; however, further studies are warranted [109].

\section{Neurodegenerative Disorders}

6.1. Overview. There is evidence that NAC may be protective for neurodegenerative disorders like Parkinson's disease, Alzheimer's disease, neuropathic pain, stroke, and multiple sclerosis (MS). As a glutathione precursor with antioxidant and anti-inflammatory properties, it may be helpful as an adjuvant for these conditions $[24,110]$.

6.2. Parkinson's Disease $(L O E=B)$. Dopamine may trigger apoptosis in neuronal cell cultures, which may initiate inappropriate loss of nigral cells in Parkinson's disease. Thiols containing compounds like NAC are markedly protective by inhibiting dopamine-induced cell death in cell cultures [111]. A clinical study using NAC both as a weekly intravenous infusion and $500 \mathrm{mg}$ orally twice a day over three months significantly improved Parkinson symptoms and increased dopamine binding in the brain warranting further study [112].

6.3. Dementia $(L O E=B)$. Animal studies have shown significant promise improving cognitive function even though beta-amyloid pathology was unchanged [113]. There is some evidence that NAC as an adjuvant may slow the progression of dementia; however, this effect was seen clinically in a nutraceutical containing several ingredients including NAC [114].

6.4. Neuropathic Pain $(L O E=A, B)$. Matrix metalloproteinases (MMPs) are one of the key components inducing neural inflammation and facilitating inflammatory cytokine maturation. N-acetylcysteine by inhibiting MMP significantly attenuates neuropathic pain in animal studies [115].

As seen above, there is benefit with NAC in diabetic neuropathy, and there is evidence that NAC at 1200 or $2400 \mathrm{mg}$ daily may reduce the incidence and severity of paclitaxel-induced peripheral neuropathy in chemotherapy treatment [116].

6.5. Stroke $(L O E=A)$. Acrolein-mediated damage after stroke has been implicated in the size of strokes in animal studies and NAC has been shown to reduce the size of the infarct [117]. In a recent randomized double-blind placebocontrolled trial using NAC 4 grams four times a day for 72 hours at the onset (within 24 hours) of an ischemic stroke, 
the follow-up National Institute of Health Stroke Scale at 90 days resulted in a better outcome profile in both neurological deficit and disability [118].

6.6. Multiple Sclerosis $(L O E=B)$. A small RCT study using intravenous NAC once weekly along with $500 \mathrm{mg}$ twice a day for two months showed improvement in glucose metabolism in several areas of the brain as well as improved attention and cognition in self-reported scores in the treatment group [119]. In a small study in progressive multiple sclerosis (MS), NAC at a dose of $1250 \mathrm{mg}$ three times a day was well tolerated and resulted in sustained fatigue improvement [120]. Glutathione levels are reduced in secondary progressive MS and NAC is able to improve this [121].

\section{Eye Conditions}

7.1. Age-Related Macular Degeneration. The addition of NAC to cell cultures of retinal pigment epithelium resulted in significant reduction of oxidative damage in cell studies [122]. NAC also upregulates reduced glutathione production and also reverses lipid peroxidation in these cells [123]. This has been suggested as a novel new treatment for macular degeneration and clinical studies are warranted.

7.2. Glaucoma $(L O E=B)$. At present, there are animal studies that suggest that NAC may decrease retinal damage caused by ocular hypertension [124]. Oxidative stress and autophagy were suppressed by NAC, which increases glutathione, suggesting that this may be useful in some types of glaucoma patients [125].

7.3. Sjogren's Syndrome (Dry Eyes) $(L O E=A)$. Oral NAC used in a double-blind study has shown improvements in daytime thirst, ocular soreness, ocular irritability, and halitosis in Sjogren's syndrome [126].

\section{Psychiatric Conditions}

There may be therapeutic benefit with NAC on schizophrenia, bipolar illness, and obsessive compulsive disorder as well as other impulsive or compulsive behaviours such as seen in gambling, substance misuse, pathological nail biting, and trichotillomania [127]. A more recent review of current evidence is in agreement with the effect of NAC on various psychiatric conditions [128]. One of the mechanisms for the benefit is improvement on mitochondrial resilience against stress [12]. With regard to addiction behaviour, NAC may provide enhanced glutamate homeostasis and modulate glutamatergic dysfunction [129].

8.1. Schizophrenia $(L O E=A)$. In schizophrenia, there is glutathione dysregulation, which improves with NAC as a precursor of glutathione [130]. There is evidence in a systematic review that NAC used as adjuvant therapy improves schizophrenia symptoms and may also improve one cognitive domain in the area of working memory [131]. The improvement was seen with the longer intervention.

8.2. Obsessive Compulsive Disorder $(L O E=A)$. An RCT using $\mathrm{NAC}$ as an adjuvant with the use of fluoxetine showed significant improvement in the NAC group in the treatment of moderate-to-severe obsessive compulsive disorder (OCD) [132]. Another RCT using NAC as an adjuvant with the use of citalopram in children and adolescents showed significant improvement in resistance/control compulsions [133]. One systematic review of the use of NAC as an adjuvant suggests that results remain inconclusive; however, because of the relative benign side-effect profile, larger, more robust studies need to be done to determine which clinical populations would benefit from this [134]. NAC used as adjuvant in treatment of resistant OCD showed a reduction in anxiety symptoms [135].

8.3. Bipolar Illness $(L O E=A)$. A major issue in bipolar disorder is treatment-resistant subthreshold depression. An RCT $(n=75)$ using NAC as augmentation strategy was found to be safe and effective for the depressive symptoms in bipolar disorder [136].

8.4. Trichotillomania, Pathologic Nail Biting, and Skin Picking $(L O E=A, B)$. Trichotillomania has been difficult to treat, and the use of serotonergic medications has been conflicting. An RCT trial using NAC for treatment of trichotillomania showed significant benefit [137]. There are a number of case studies and short trials that show benefit in pathologic nail biting [138] and skin picking [139].

8.5. Addiction Behaviour $(L O E=B)$. In cocaine-seeking behaviour, NAC was useful in reducing relapse by providing glutamate homeostasis in animal studies [140]. In humans, reduced cravings in substance use disorders have been seen in some early studies with the use of NAC [141]. This was seen in most but not all studies in cannabis use disorder, alcohol use disorder, and smoking use disorder.

8.6. Use as Chelator for Metal Toxicity $(L O E=A, B)$. $\mathrm{N}$-acetylcysteine has been shown to chelate toxic metals in animal studies as well as in human studies with little or no effect on essential metals. Mercury, lead, gold, and arsenic have been removed in humans although the studies are limited. The evidence for removal of lead is more robust because of a double-blind placebo-controlled trial [142]. Metal on metal hip prosthesis often results in increased chromium in the blood and NAC has been helpful in reducing levels safely [143]. Adverse effects of arsenic-induced hepatotoxicity in rats were countered by NAC [144]. In a case report of acute ingestion of a potentially lethal overdose of sodium arsenate ant poison, intravenous NAC reversed the clinical outcome of expected death [145]. 
8.7. Nanoparticle-Induced Reduction of Deoxyribonucleic Acid Methylation $(L O E=B)$. There has been increasing concern with the toxicity of nanoparticles causing cellular damage by increasing reactive oxygen species as an epigenetic mechanism decreasing deoxyribonucleic acid (DNA) methylation. Preclinical studies show that NAC reverses and prevents the oxidative damage caused by engineered nanoparticles [146].

8.8. Side Effects. One of the properties of NAC is that it has an unpleasant smell and taste but is generally well tolerated in oral doses below $1200 \mathrm{mg} /$ day. It may also cause some nausea vomiting and diarrhea. Vomiting after intravenous use has been reported in about $11 \%$ at doses of $150 \mathrm{mg} / \mathrm{kg}$ and one anaphylactic reaction has been reported [147]. $\mathrm{N}$-acetylcysteine has anticoagulant and platelet inhibiting properties and the use in patients with bleeding disorders or blood thinners may be relatively counterindicated [148]. The use of NAC with patients on nitroglycerine should be cautioned, since it may cause hypotension [149]. Other more rare side effects may include stomatitis, drowsiness, rhinorrhea, and hemoptysis [127].

8.9. Dosing of NAC. N-acetylcysteine dosing varies significantly with various clinical studies and doses of $1200 \mathrm{mg}$ daily or more are usually required to be clinically relevant. Studies in metabolic diseases show that 5-600 mg orally per day may be sufficient to ameliorate fatty liver disease. For Crohn's disease and ulcerative colitis, doses of $800 \mathrm{mg}$ per day seemed sufficient. Doses as high as $1250 \mathrm{mg}$ orally three times a day have been used safely in MS and showed benefit in reducing fatigue. Doses of $8000 \mathrm{mg} /$ day orally did not cause clinically significant reactions in HIV patients [150]. Clearly dosing is still debated and much needs to be learned in this area.

\section{Discussion and Conclusion}

$\mathrm{N}$-acetylcysteine appears to be well tolerated with minimal side effects when used as a supplement or in treatment of various disorders. As stated above, the dosage required for this medication is not always clear, and there is much work needed to provide this information. A number of mechanisms of its actions are listed in Table 1. These actions provide reasoning for some of the results that are seen in so many different conditions. Many other conditions not listed in this document are emerging as understanding about NAC grows. As seen with other antioxidants in the past, there is some caution as expressed above with lung cancer models, where there may be an increase in proliferation as a result of p53 inhibition or likewise a possible increase in pulmonary hypertension.

As seen above, benefit has been shown with pulmonary, psychiatric, neurologic, metabolic, and infectious diseases, fertility issues, and some cancers. For most of these conditions, NAC can be used as an adjuvant, which may improve quality of life, morbidity, and mortality.
The use in metal toxicity and recent evidence in protecting DNA are also important. Much needs to be learned and more in vivo studies need to be performed to give us more confidence in using this simple compound.

\section{Data Availability}

The data used to support the findings of this study are available on PubMed.

\section{Conflicts of Interest}

The author declares that there are no conflicts of interest.

\section{References}

[1] C. B. Lillibridge, J. M. Docter, and S. Eidelman, "Oral administration of $\mathrm{n}$-acetyl cysteine in the prophylaxis of "meconium ileus equivalent," The Journal of Pediatrics, vol. 71, no. 6, pp. 887-889, 1967.

[2] M. Gracey, V. Burke, and C. M. Anderson, "Treatment of abdominal pain in cystic fibrosis by oral administration of n-acetyl cysteine," Archives of Disease in Childhood, vol. 44, no. 235, pp. 404-405, 1969.

[3] S. Vasdev, P. Singal, and V. Gill, "The antihypertensive effect of cysteine," The International Journal of Angiology: Official Publication of the International College of Angiology, Inc, vol. 18, no. 1, pp. 7-21, 2009.

[4] O. Demirkol, C. Adams, and N. Ercal, "Biologically important thiols in various vegetables and fruits," Journal of Agricultural and Food Chemistry, vol. 52, no. 26, pp. 81518154, 2004.

[5] C. Kerksick and D. Willoughby, "The antioxidant role of glutathione and N-Acetyl-Cysteine supplements and exercise-induced oxidative stress," Journal of the International Society of Sports Nutrition, vol. 2, no. 2, p. 38, 2005.

[6] D. Fass and C. Thorpe, "Chemistry and enzymology of disulfide cross-linking in proteins," Chemical Review, vol. 118, no. 3, pp. 1169-1198, 2018.

[7] S. De Flora, A. Izzotti, F. D'Agostini, and R. Balansky, "Mechanisms of $\mathrm{N}$-acetylcysteine in the prevention of DNA damage and cancer, with special reference to smoking-related end-points," Carcinogenesis, vol. 22, no. 7, pp. 999-1013, 2001.

[8] M. Halasi, M. Wang, T. S. Chavan, V. Gaponenko, N. Hay, and A. L. Gartel, "ROS inhibitor N-acetyl-L-cysteine antagonizes the activity of proteasome inhibitors," Biochemical Journal, vol. 454, no. 2, pp. 201-208, 2013.

[9] S. Uraz, "N-acetylcysteine expresses powerful anti-inflammatory and antioxidant activities resulting in complete improvement of acetic acid-induced colitis in rats," Scandinavian Journal of Clinical and Laboratory Investigation, vol. 73, no. 1, pp. 61-66, 2013.

[10] A. Zhitkovich, "N-Acetylcysteine: Antioxidant, aldehyde scavenger, and more," Chemical Research in Toxicology, vol. 32, no. 7, pp. 1318-1319, 2019.

[11] C. R. Livingstone, M. A. Andrews, S. M. Jenkins, and C. Marriott, "Model systems for the evaluation of mucolytic drugs: acetylcysteine and S-carboxymethylcysteine," Journal of Pharmacy and Pharmacology, vol. 42, no. 2, pp. 73-78, 1990.

[12] G. R. Fries and F. Kapczinski, "N-acetylcysteine as a mitochondrial enhancer: a new class of psychoactive drugs?" Brazilian Journal of Psychiatry, vol. 33, pp. 321-322, 2011. 
[13] Y. Jiao, "N-acetyl cysteine depletes reactive oxygen species and prevents dental monomer-induced intrinsic mitochondrial apoptosis in vitro in human dental pulp cells," PLOS ONE, vol. 11, no. 1, Article ID e0147858, 2016.

[14] D. A. Rossignol, "The use of N-acetylcysteine as a chelator for metal toxicity," in The Therapeutic Use of $N$-Acetylcysteine (NAC) in Medicine, R. E. Frye and M. Berk, Eds., Springer Singapore, Singapore, pp. 169-179, 2019.

[15] O. Dean, F. Giorlando, and M. Berk, "N-acetylcysteine in psychiatry: current therapeutic evidence and potential mechanisms of action," J Psychiatry Neurosci, vol. 36, no. 2, pp. 78-86, 2011.

[16] M. G. Tomàs Casanova, "N-Acetylcysteine: An old drug with variable anti-influenza properties," Journal of Controversies in Biomedical Research, vol. 2, no. 1, pp. 1-8, 2016.

[17] P. Redondo, E. Jimenez, A. Perez, and J. García-Foncillas, "N-acetylcysteine downregulates vascular endothelial growth factor production by human keratinocytes in vitro," Archives of Dermatological Research, vol. 292, no. 12, pp. 621-628, 2000.

[18] L. Douiev, D. Soiferman, C. Alban, and A. Saada, "The effects of ascorbate, $\mathrm{N}$-acetylcysteine, and resveratrol on fibroblasts from patients with mitochondrial disorders," Journal of Clinical Medicine, vol. 6, no. 1, 2016.

[19] V. Martina, "Long-term N-acetylcysteine and L-arginine administration reduces endothelial activation and systolic blood pressure in hypertensive patients with type 2 diabetes," Diabetes Care, vol. 31, no. 5, pp. 940-944, 2008.

[20] B. Olsson, "Pharmacokinetics and bioavailability of reduced and oxidized N-acetylcysteine," European Journal of Clinical Pharmacology, vol. 34, no. 1, pp. 77-82, 1988.

[21] L. Borgström, B. Kågedal, and O. Paulsen, "Pharmacokinetics of N-acetylcysteine in man," European Journal of Clinical Pharmacology, vol. 31, no. 2, pp. 217-222, 1986.

[22] M. R. Holdiness, "Clinical pharmacokinetics of N-acetylcysteine," Clinical Pharmacokinetics, vol. 20, no. 2, pp. 123-134, 1991.

[23] W. Droge, "Oxidative stress and ageing: is ageing a cysteine deficiency syndrome?" Philosophical Transactions of the Royal Society B: Biological Sciences, vol. 360, no. 1464, pp. 2355-2372, 2005.

[24] Deepmala, N. Kumar, M. Berk et al., "Clinical trials of $\mathrm{N}$-acetylcysteine in psychiatry and neurology: a systematic review," Neuroscience and Biobehavioral Reviews, vol. 55, pp. 294-321, 2015.

[25] P. V. Dludla, L. Tiano, J. Louw et al., "The beneficial effects of $\mathrm{N}$-acetyl cysteine (NAC) against obesity associated complications: a systematic review of pre-clinical studies," Pharmacological Research, vol. 146, Article ID 104332, 2019.

[26] K. Fowdar, H. Chen, Z. He et al., "The effect of N-acetylcysteine on exacerbations of chronic obstructive pulmonary disease: a meta-analysis and systematic review," Heart and Lung, vol. 46, no. 2, pp. 120-128, 2017.

[27] Z. Shi and C. A. Puyo, "N-acetylcysteine to combat COVID19: an evidence review," Therapeutics and Clinical Risk Management, vol. 16, pp. 1047-1055, 2020.

[28] N. Devi and C. Boya, D. Bansal, N-acetyl-cysteine as adjuvant therapy in female infertility: a systematic review and meta-analysis," Journal of Basic and Clinical Physiology and Pharmacology, 2020.

[29] M. H. Ebell, J. Siwek, B. D. Weiss et al., "Strength of recommendation taxonomy (SORT): a patient-centered approach to grading evidence in the medical literature," American Family Physician, vol. 69, no. 3, pp. 548-556, 2004.
[30] R. Tirouvanziam, C. K. Conrad, T. Bottiglieri et al., "Highdose oral $\mathrm{N}$-acetylcysteine, a glutathione prodrug, modulates inflammation in cystic fibrosis," Proceedings of the National Academy of Sciences of the United States of America, vol. 103, no. 12, pp. 4628-4633, 2006.

[31] C. Conrad, J. Lamp, C. Dunn et al., "Long-term treatment with oral $\mathrm{N}$-acetylcysteine: affects lung function but not sputum inflammation in cystic fibrosis subjects. A phase II randomized placebo-controlled trial," Journal of Cystic Fibrosis, vol. 14, no. 2, pp. 219-227, 2015.

[32] Y. C. Duijvestijn and P. L. Brand, "Systematic review of $\mathrm{N}$-acetylcysteine in cystic fibrosis," Acta Paediatrica, vol. 88, no. 1, pp. 38-41, 1999.

[33] J. McNamara, L. Zhang, and N. Demirel, “The effect of long term use of inhaled $\mathrm{N}$-acetylcysteine on cystic fibrosis lung disease," C67. Suppurative Lung Diseases In Children, Article ID A6142, 2017.

[34] A. L. Schauble, E. J. Bisaccia, G. Lee, and S. Z. Nazr, "Nacetylcysteine for management of distal intestinal obstruction syndrome," The Journal of Pediatric Pharmacology and Therapeutics: JPPT: The Official Journal of PPAG, vol. 24, no. 5, pp. 390-397, 2019.

[35] L. M. Simon and N. Suttorp, "Lung cell oxidant injury: decrease in oxidant mediated cytotoxicity by $\mathrm{N}$-acetylcysteine," European Journal of Respiratory Diseases. Supplement, vol. 139, pp. 132-135, 1985.

[36] M. Cazzola, L. Calzatta, C. Page et al., "Influence of $\mathrm{N}$-acetylcysteine on chronic bronchitis or COPD exacerbations: a meta-analysis," European Respiratory Review, vol. 24, no. 137, pp. 451-461, 2015.

[37] C. Stey, J. Steurer, S. Bachmann, T. C. Medici, and M. R. Tramèr, "The effect of oral N-acetylcysteine in chronic bronchitis: a quantitative systematic review," European Respiratory Journal, vol. 16, no. 2, pp. 253-262, 2000.

[38] C. M. Sanguinetti, "N-acetylcysteine in COPD: why, how, and when?" Multidisciplinary Respiratory Medicine, vol. 11, p. 8, 2015.

[39] M. Aliyali, A. Poorhasan Amiri, A. Sharifpoor, and F. Zalli, "Effects of N-acetylcysteine on asthma exacerbation," Iranian Journal of Allergy, Asthma and Immunology, vol. 9, no. 2, pp. 103-109, 2010.

[40] P. Eftekhari, J. J. Li, M. Yang et al., "Preventive effect of $\mathrm{N}$-acetylcysteine in a mouse model of steroid resistant acute exacerbation of asthma," EXCLI Journal, vol. 12, pp. 184-192, 2013.

[41] G. V. Guibas, E. Spandou, S. Meditskou, T. A. Vyzantiadis, K. N. Priftis, and G. Anogianakis, "N-acetylcysteine exerts therapeutic action in a rat model of allergic rhinitis," International Forum of Allergy and Rhinology, vol. 3, no. 7, pp. 543-549, 2013.

[42] C. J. Lane, D. Redding, K. A. Gonzalez, V. Cardenas, I. Boldogh, and S. Sur, "Topical N-acetyl cysteine (NAC) reduces late phase nasal symptoms following ragweed challenge," Journal of Allergy and Clinical Immunology, vol. 123, no. 2, p. S53, 2009.

[43] F. Blasi, C. Page, M. Cazzola et al., "The effect of N-acetylcysteine on biofilms: implications for the treatment of respiratory tract infections," Respiratory Medicine, vol. 117, pp. 190-197, 2016.

[44] Q. Qi, C. Li, Y. Li et al., "Effect of N-acetylcysteine on exacerbations of bronchiectasis (BENE): a randomized controlled trial," Respiratory Research, vol. 20, no. 1, p. 73, 2019.

[45] F. Naz, A. B. Raza, I. Ijaz, and M. Y. Kazi, "Effectiveness of nebulized $\mathrm{N}$-acetylcysteine solution in children with acute 
bronchiolitis," Journal of College of Physicians and Surgeons Pakistan, vol. 24, no. 6, pp. 408-411, 2014.

[46] "Prednisone, Azathioprine and N-acetylcysteine for pulmonary fibrosis," New England Journal of Medicine, vol. 366, no. 21, pp. 1968-1977, 2012.

[47] F. Fen, J. Zhang, Z. Wang, Q. Wu, and X. Zhou, "Efficacy and safety of $\mathrm{N}$-acetylcysteine therapy for idiopathic pulmonary fibrosis: an updated systematic review and meta-analysis," Experimental and Therapeutic Medicine, vol. 18, no. 1, pp. 802-816, 2019.

[48] M. J. Smilkstein, G. L. Knapp, K. W. Kulig, and B. H. Rumack, "Efficacy of oral N-acetylcysteine in the treatment of acetaminophen overdose. Analysis of the national multicenter study (1976 to 1985)," New England Journal of Medicine, vol. 319, no. 24, pp. 1557-1562, 1988.

[49] R. G. Peterson and B. H. Rumack, "N-acetylcysteine for acetaminophen overdosage (cont.)," New England Journal of Medicine, vol. 296, no. 9, p. 515, 1977.

[50] M. R. Smith and R. L. Davis, "Mycetismus: a review," Gastroenterology Report (Oxf), vol. 4, no. 2, pp. 107-112, 2016.

[51] J. N. Drault, E. Baelen, H. Mehdaoui, J. M. Delord, and F. Flament, "Massive paraquat poisoning. Favorable course after treatment with n-acetylcysteine and early hemodialysis," Annales Françaises d'Anesthésie et de Réanimation, vol. 18, no. 5, pp. 534-537, 1999.

[52] D. M. Dell'Aglio, M. E. Sutter, M. D. Schwartz, D. D. Koch, D. A. Algren, and B. W. Morgan, "Acute chloroform ingestion successfully treated with intravenously administered $\mathrm{N}$-acetylcysteine," Journal of Medical Toxicology, vol. 6, no. 2, pp. 143-146, 2010.

[53] I. K. Lai, K. Dhakal, G. S. Gadupudi et al., "N-acetylcysteine (NAC) diminishes the severity of PCB 126-induced fatty liver in male rodents," Toxicology, vol. 302, no. 1, pp. 25-33, 2012.

[54] P. M. Harrison, J. A. Wendon, and A. E. S. Gimson, "Improvement by acetylcysteine of hemodynamics and oxygen transport in fulminant hepatic failure," New England Journal of Medicine, vol. 324, no. 26, pp. 1852-1857, 1991.

[55] T. Nabi et al., "Role of N-acetylcysteine treatment in nonacetaminophen-induced acute liver failure: a prospective study," Saudi Journal of Gastroenterology, vol. 23, no. 3, pp. 169-175, 2017.

[56] K. Mumtaz, S. Nabi, N. Rafiq, and A. Shah, "Role of $\mathrm{N}$-acetylcysteine in adults with non-acetaminophen-induced acute liver failure in a center without the facility of liver transplantation," Hepatology International, vol. 3, no. 4, pp. 563-570, 2009.

[57] J. Hu, "Efficacy and safety of acetylcysteine in "non-acetaminophen" acute liver failure: a meta-analysis of prospective clinical trials," Clinics and Research in Hepatology and Gastroenterology, vol. 39, no. 5, pp. 594-599, 2015.

[58] N. A. Kretzmann, E. Chiela, U. Matte, N. Marroni, and C. A. Marroni, "N-acetylcysteine improves antitumoural response of Interferon alpha by NF-kB downregulation in liver cancer cells," Comparative Hepatology, vol. 11, no. 1, p. 4, 2012.

[59] A. R. Bourgonje, G. Dijkstra, H. V. Goor et al., "Crohn's disease in clinical remission is marked by systemic oxidative stress," Frontiers in Physiology, vol. 10, no. 499, 2019.

[60] S. Kourosh Masnadi, S. Sotoudeh, A. M. Shirazi, S. -Y. Moaddab, Z. Nourpanah, and Z. Nikniaz, Effect of NAcetylcysteine on Remission Maintenance in Patients with
Ulcerative Colitis: A Randomized Controlled Clinical Trial, Research Square, Durham, NC, USA, 2021.

[61] F. A. Moura, K. Q. D. Andrade, J. C. F. D. Santos, O. R. P. Araújo, and M. O. F. Goulart, "Antioxidant therapy for treatment of inflammatory bowel disease: does it work?" Redox Biology, vol. 6, pp. 617-639, 2015.

[62] A. O’Loghlen, "N-acetyl-cysteine abolishes hydrogen peroxide-induced modification of eukaryotic initiation factor $4 \mathrm{~F}$ activity via distinct signalling pathways," Cell Signal, vol. 18, no. 1, pp. 21-31, 2006.

[63] Z. W. Lai, R. Hanczko, E. Bonilla, and T. N. Caza, "Nacetylcysteine reduces disease activity by blocking mammalian target of rapamycin in T cells from systemic lupus erythematosus patients: a randomized, double-blind, placebo-controlled trial," Arthritis and Rheumatology, vol. 64, no. 9, pp. 2937-2946, 2012.

[64] P. V. Dludla, J. Louw, L. Tiano et al., "N-acetyl cysteine targets hepatic lipid accumulation to curb oxidative stress and inflammation in NAFLD: a comprehensive analysis of the literature," Antioxidants (Basel), vol. 9, no. 12, 2020.

[65] A. Falach-Malik, H. Rozenfeld, M. Chetboun et al., "NAcetyl-L-Cysteine inhibits the development of glucose intolerance and hepatic steatosis in diabetes-prone mice," American Journal of Translational Research, vol. 8, no. 9, pp. 3744-3756, 2016

[66] A. K. Kaga, P. O. Barbanera, N. O. L. D. Carmo et al., "Effect of $\mathrm{N}$-acetylcysteine on dyslipidemia and carbohydrate metabolism in STZ-induced diabetic rats," International Journal of Vascular Medicine, vol. 2018, Article ID 6428630, 7 pages, 2018.

[67] M. A. Szkudlinska, A. D. von Frankenberg, and K. M. Utzschneider, "The antioxidant N-Acetylcysteine does not improve glucose tolerance or $\beta$-cell function in type 2 diabetes," Journal of Diabetes and its Complications, vol. 30, no. 4, pp. 618-622, 2016.

[68] M. Sagara, "Inhibition of development of peripheral neuropathy in streptozotocin-induced diabetic rats with N-acetylcysteine," Diabetologia, vol. 39, no. 3, pp. 263-269, 1996.

[69] Y. Zhu, X.-L. Zhang, B.-F. Zhu, and Y.-N. Ding, "Effect of antioxidant $\mathrm{N}$-acetylcysteine on diabetic retinopathy and expression of VEGF and ICAM-1 from retinal blood vessels of diabetic rats," Molecular Biology Reports, vol. 39, no. 4, pp. 3727-3735, 2012.

[70] G. B. Nogueira, G. R. Punaro, C. S. Oliveira et al., "N-acetylcysteine protects against diabetic nephropathy through control of oxidative and nitrosative stress by recovery of nitric oxide in rats," Nitric Oxide, vol. 78, pp. 22-31, 2018.

[71] N. Heidari, F. Sajedi, Y. Mohammadi, M. Mirjalili, and M. Mehrpooya, "Ameliorative effects of $\mathrm{N}$-acetylcysteine as adjunct therapy on symptoms of painful diabetic neuropathy," Journal of Pain Research, vol. 12, pp. 3147-3159, 2019.

[72] A. M. Fulghesu, "N-acetyl-cysteine treatment improves insulin sensitivity in women with polycystic ovary syndrome," Fertility and Sterility, vol. 77, no. 6, pp. 1128-1135, 2002.

[73] D. Thakker, A. Raval, I. Patel, and R. Walia, "N-acetylcysteine for polycystic ovary syndrome: a systematic review and meta-analysis of randomized controlled clinical trials," Obstetrics and Gynecology International, vol. 2015, Article ID 817849, 13 pages, 2015.

[74] D. D. Jenkins, "Fetal and neonatal effects of $\mathrm{N}$-acetylcysteine when used for neuroprotection in maternal chorioamnionitis," The Journal of Pediatrics, vol. 168, pp. 67-76.e6, 2016. 
[75] A. F. Amin, O. M. Shaaban, and M. A. Bediawy, "N-acetyl cysteine for treatment of recurrent unexplained pregnancy loss," Reproductive Biomedicine Online, vol. 17, no. 5, pp. 722-726, 2008.

[76] H. Ciftci, A. Verit, M. Savas, E. Yeni, and O. Erel, "Effects of $\mathrm{N}$-acetylcysteine on semen parameters and oxidative/antioxidant status," Urology, vol. 74, no. 1, pp. 73-76, 2009.

[77] M. R. Safarinejad and S. Safarinejad, "Efficacy of selenium and/or N-acetyl-cysteine for improving semen parameters in infertile men: a double-blind, placebo controlled, randomized study," J Urol, vol. 181, no. 2, pp. 741-751, 2009.

[78] M. Renke, "The effect of N-acetylcysteine on blood pressure and markers of cardiovascular risk in non-diabetic patients with chronic kidney disease: a placebo-controlled, randomized, cross-over study," Medical Science Monitor, vol. 16, no. 7, pp. Pi13-8, 2010.

[79] W. Hildebrandt, R. Saucer, K. A. Dugi et al., "Oral N-acetylcysteine reduces plasma homocysteine concentrations regardless of lipid or smoking status," The American Journal of Clinical Nutrition, vol. 102, no. 5, pp. 1014-1024, 2015.

[80] W. Yu, X. Song, C. Lin, and W. Ji, "Interventions and mechanisms of $\mathrm{N}$-acetylcysteine on monocrotaline-induced pulmonary arterial hypertension," Experimental and Therapeutic Medicine, vol. 15, no. 6, pp. 5503-5509, 2018.

[81] M. C. Chaumais, "N-acetylcysteine improves established monocrotaline-induced pulmonary hypertension in rats," Respiratory Research, vol. 15, no. 1, p. 65, 2014.

[82] L. A. Palmer, A. Doctor, P. Chhabra et al., "S-nitrosothiols signal hypoxia-mimetic vascular pathology," Journal of Clinical Investigation, vol. 117, no. 9, pp. 2592-2601, 2007.

[83] T. Nishikawa, "Mechanisms of fatal cardiotoxicity following high-dose cyclophosphamide therapy and a method for its prevention," PLoS One, vol. 10, no. 6, Article ID e0131394, 2015.

[84] K. A. Mills, R. Chess-Williams, and C. McDermott, "Novel insights into the mechanism of cyclophosphamide-induced bladder toxicity: chloroacetaldehyde's contribution to urothelial dysfunction in vitro," Archives of Toxicology, vol. 93, no. 11, pp. 3291-3303, 2019.

[85] S. Huang, J. You, Q. Wang et al., "N-acetylcysteine attenuates cisplatin-induced acute kidney injury by inhibiting the C5a receptor," Biomed Research International, vol. 2019, Article ID 4805853, 11 pages, 2019.

[86] L. L. Muldoon, "N-acetylcysteine chemoprotection without decreased cisplatin antitumor efficacy in pediatric tumor models," Journal of Neuro-Oncology, vol. 121, no. 3, pp. 433-440, 2015.

[87] D. Monti, F. Sotgia, P. Cotzia et al., "Pilot study demonstrating metabolic and anti-proliferative effects of in vivo anti-oxidant supplementation with $\mathrm{N}$-Acetylcysteine in Breast Cancer," Seminars in Oncology, vol. 44, no. 3, pp. 226-232, 2017.

[88] Y. J. Lee, D. M. Lee, C. H. Lee et al., "Suppression of human prostate cancer PC-3 cell growth by N-acetylcysteine involves over-expression of Cyr61," Toxicology In Vitro, vol. 25, no. 1, pp. 199-205, 2011.

[89] V. I. Sayin, "Antioxidants accelerate lung cancer progression in mice," Science Translation Medicine, vol. 6, no. 221, p. 221ra15, 2014.

[90] J. D. Lambert, S. Sang, and C. S. Yang, "N-Acetylcysteine enhances the lung cancer inhibitory effect of epigallocatechin-3-gallate and forms a new adduct," Free Radical Biology and Medicine, vol. 44, no. 6, pp. 1069-1074, 2008.
[91] S. Rakshit, J. Bagchi, L. Mandal et al., "N-acetyl cysteine enhances imatinib-induced apoptosis of Bcr-Abl+ cells by endothelial nitric oxide synthase-mediated production of nitric oxide," Apoptosis, vol. 14, no. 3, pp. 298-308, 2009.

[92] J. Deng, "N-acetylcysteine decreases malignant characteristics of glioblastoma cells by inhibiting Notch2 signaling," Journal of Experimental and Clinical Cancer Research, vol. 38, no. 1, p. 2, 2019.

[93] M. F. Mark F McCarty and J. H. O’Keefe, "NADPH oxidase, uncoupled endothelial nitric oxide synthase, and NF-KappaB are key mediators of the pathogenic impact of obstructive sleep apnea - therapeutic implications," Journal of Integrative Cardiology, vol. 2, no. 5, pp. 367-374, 2016.

[94] K. Sadasivam, K. Patial, V. K. Vijayan, and K. Ravi, "Antioxidant treatment in obstructive sleep apnoea syndrome," Indian Journal of Chest Diseases and Allied Sciences, vol. 53, no. 3, pp. 153-162, 2011.

[95] K. Wu, X. Su, G. Li, and N. Zhang, "Antioxidant carbocysteine treatment in obstructive sleep apnea syndrome: a randomized clinical trial," PLoS One, vol. 11, no. 2, Article ID e0148519, 2016.

[96] L. Arranz, "The glutathione precursor N-acetylcysteine improves immune function in postmenopausal women," Free Radical Biology and Medicine, vol. 45, no. 9, pp. 1252-1262, 2008.

[97] B. Akerlund, C. Jarstrand, B. Lindeke, A. Sönnerborg, A. C. Akerblad, and O. Rasool, "Effect of N-acetylcysteine(NAC) treatment on HIV-1 infection: a double-blind placebo-controlled trial," European Journal of Clinical Pharmacology, vol. 50, no. 6, pp. 457-461, 1996.

[98] M. Roederer, S. W. Ela, F. J. Staal, and L. A. Herzenberg, "Nacetylcysteine: a new approach to anti-HIV therapy," AIDS Research and Human Retroviruses, vol. 8, no. 2, pp. 209-217, 1992.

[99] S. M. Mahakalkar, D. Nagrale, S. Gaur, C. Urade, B. Murhar, and A. Turankar, "N-acetylcysteine as an add-on to Directly Observed Therapy Short-I therapy in fresh pulmonary tuberculosis patients: a randomized, placebo-controlled, double-blinded study," Perspectives in Clinical Research, vol. 8, no. 3, pp. 132-136, 2017.

[100] T. Jeeraaumponwat, "N-acetylcysteine and mortality in hospitalized pulmonary tuberculosis infection," European Respiratory Journal, vol. 54, no. suppl 63, p. PA2958, 2019.

[101] J. Geiler, H.-W. Doerr, J. Cinatl et al., "N-acetyl-L-cysteine (NAC) inhibits virus replication and expression of pro-inflammatory molecules in A549 cells infected with highly pathogenic $\mathrm{H} 5 \mathrm{~N} 1$ influenza A virus," Biochemical Pharmacology, vol. 79, no. 3, pp. 413-420, 2010.

[102] M. Mata, "N-acetyl-L-cysteine (NAC) inhibit mucin synthesis and pro-inflammatory mediators in alveolar type II epithelial cells infected with influenza virus $A$ and $B$ and with respiratory syncytial virus (RSV)," Biochemical Pharmacology, vol. 82, no. 5, pp. 548-555, 2011.

[103] S. De Flora, R. Balansky, and S. La Maestra, "Rationale for the use of $\mathrm{N}$-acetylcysteine in both prevention and adjuvant therapy of COVID-19," The FASEB Journal, vol. 34, no. 10, pp. 13185-13193, 2020.

[104] R. M. Jorge-Aarón and M. P. Rosa-Ester, "N-acetylcysteine as a potential treatment for COVID-19," Future Microbiology, vol. 15, pp. 959-962, 2020.

[105] Y. Liu, M. Wang, G. Liu et al., "Experience of N-acetylcysteine airway management in the successful treatment of one case of critical condition with COVID-19: a case report," Medicine, vol. 99, no. 42, Article ID e22577, 2020. 
[106] A. K. Gurbuz, "Effect of N-acetyl cysteine on Helicobacter pylori," Southern Medical Journal, vol. 98, no. 11, pp. 1095-1097, 2005.

[107] K. Makipour and F. K. Friedenberg, "The potential role of $\mathrm{N}$-acetylcysteine for the treatment of Helicobacter pylori," Journal of Clinical Gastroenterology, vol. 45, no. 10, pp. 841-843, 2011.

[108] S. Jang, E. J. Bak, and J. H. Cha, "N-acetylcysteine prevents the development of gastritis induced by Helicobacter pylori infection," Journal of Microbiology, vol. 55, no. 5, pp. 396-402, 2017.

[109] L. E. S. Fontes, A. L. C. Martimbianco, C. Zanin, and R. Riera, "N-acetylcysteine as an adjuvant therapy for Helicobacter pylori eradication," Cochrane Database Systematic Review, vol. 2, Article ID CD012357, 2019.

[110] G. Tardiolo, P. Bramanti, and E. Mazzon, "Overview on the effects of $\mathrm{N}$-acetylcysteine in neurodegenerative diseases," Molecules, vol. 23, no. 12, 2018.

[111] D. Offen, I. Ziv, H. Sternin et al., "Prevention of dopamineinduced cell death by thiol antioxidants: possible implications for treatment of Parkinson's disease," Experimental Neurology, vol. 141, no. 1, pp. 32-39, 1996.

[112] D. A. Monti, G. Zabrecky, D. Kremens et al., "N-acetyl cysteine is associated with dopaminergic improvement in Parkinson's disease," Clinical Pharmacology \& Therapeutics, vol. 106, no. 4, pp. 884-890, 2019.

[113] T. Joy, M. S. Rao, S. Madhyastha, and K. Pai, "Effect of $\mathrm{N}$-acetyl cysteine on intracerebroventricular colchicine induced cognitive deficits, beta amyloid pathology, and glial cells," Neuroscience Journal, vol. 2019, Article ID 7547382, 13 pages, 2019.

[114] Y. Hara, "Evaluation of the neuroprotective potential of $\mathrm{N}$-acetylcysteine for prevention and treatment of cognitive aging and dementia," Journal of Prevention of Alzheimer'Disease, vol. 4, no. 3, pp. 201-206, 2017.

[115] J. Li, L. Xu, X. Deng et al., "N-acetyl-cysteine attenuates neuropathic pain by suppressing matrix metalloproteinases," Pain, vol. 157, no. 8, pp. 1711-1723, 2016.

[116] H. G. Khalefa, M. A. Shawki, R. Aboelhassan, and L. M. E. Wakeel, "Evaluation of the effect of $\mathrm{N}$-acetylcysteine on the prevention and amelioration of paclitaxel-induced peripheral neuropathy in breast cancer patients: a randomized controlled study," Breast Cancer Research and Treatment, vol. 183, no. 1, pp. 117-125, 2020.

[117] T. Uemura, "Protective effects of brain infarction by N-acetylcysteine derivatives," Stroke, vol. 49, no. 7, pp. 1727-1733, 2018.

[118] M. Sabetghadam, P. Abolfathi, Y. Mohammadi, and M. Mehrpooya, "Evidence for a beneficial effect of oral $\mathrm{N}$-acetylcysteine on functional outcomes and inflammatory biomarkers in patients with acute ischemic stroke," Neuropsychiatric Disease and Treatment, no. 16, pp. 1265-1278, 2020.

[119] D. A. Monti, G. Zabrecky, T. P. Leist et al., "N-acetyl cysteine administration is associated with increased cerebral glucose metabolism in patients with multiple sclerosis: an exploratory study," Frontiers in Neurology, vol. 11, p. 88, 2020.

[120] K. Krysko, "N-acetyl cysteine for fatigue in progressive multiple sclerosis: a pilot randomized double-blind placebocontrolled trial (P5.2-093)," Neurology, vol. 92, no. 15 Supplement, pp. P5-093, 2019.

[121] I. Y. Choi, S.-P. Lee, D. R. Denney, and S. G. Lynch, "Lower levels of glutathione in the brains of secondary progressive multiple sclerosis patients measured by $1 \mathrm{H}$ magnetic resonance chemical shift imaging at 3 T," Multiple Sclerosis, vol. 17, no. 3, pp. 289-296, 2011.

[122] M. R. Terluk, M. C. Ebeling, C. R. Fisher et al., "N-Acetyl-Lcysteine protects human retinal pigment epithelial cells from oxidative damage: implications for age-related macular degeneration," Oxidative Medicine and Cellular Longevity, vol. 2019, Article ID 5174957, 14 pages, 2019.

[123] A. M. Schimel, L. Abraham, D. Cox et al., "N-acetylcysteine amide (NACA) prevents retinal degeneration by up-regulating reduced glutathione production and reversing lipid peroxidation," American Journal of Pathology, vol. 178, no. 5, pp. 2032-2043, 2011.

[124] G. Ozdemir, F. I. Tolun, M. Gul, and S. Imrek, "Retinal oxidative stress induced by intraocular hypertension in rats may be ameliorated by brimonidine treatment and $\mathrm{N}$-acetyl cysteine supplementation," Journal of Glaucoma, vol. 18, no. 9, pp. 662-665, 2009.

[125] H. Sano, K. Namekata, A. Kimura et al., "Differential effects of $\mathrm{N}$-acetylcysteine on retinal degeneration in two mouse models of normal tension glaucoma," Cell Death Disease, vol. 10, no. 2, p. 75, 2019.

[126] M. T. Walters, "A double-blind, cross-over, study of oral $\mathrm{N}$-acetylcysteine in Sjögren's syndrome," Scandinavian Journal of Rheumatology, vol. 61, pp. 253-258, 1986.

[127] R. A. Sansone and L. A. Sansone, "Getting a knack for NAC: $\mathrm{N}$-Acetyl-Cysteine," Innovations in Clinical Neuroscience, vol. 8, no. 1, pp. 10-14, 2011.

[128] S. L. Ooi, R. Green, and S. C. Pak, "N-acetylcysteine for the treatment of psychiatric disorders: a review of current evidence," Biomed Research International, vol. 2018, Article ID 2469486, 8 pages, 2018.

[129] K. J. Reissner and P. W. Kalivas, "Using glutamate homeostasis as a target for treating addictive disorders," Behavioural Pharmacology, vol. 21, no. 5-6, pp. 514-522, 2010.

[130] S. Lavoie, M. M. Murray, P. Deppen et al., "Glutathione precursor, $\mathrm{N}$-acetyl-cysteine, improves mismatch negativity in schizophrenia patients," Neuropsychopharmacology, vol. 33, no. 9, pp. 2187-2199, 2008.

[131] C. O. Yolland, D. Hanratty, E. Neill et al., "Meta-analysis of randomised controlled trials with $\mathrm{N}$-acetylcysteine in the treatment of schizophrenia," Australian and New Zealand Journal of Psychiatry, vol. 54, no. 5, pp. 453-466, 2020.

[132] K. Paydary, A. Akamaloo, A. Ahmadipour et al., "N-acetylcysteine augmentation therapy for moderate-to-severe obsessive-compulsive disorder: randomized, double-blind, placebo-controlled trial," Journal of Clinical Pharmacy and Therapeutics, vol. 41, no. 2, pp. 214-219, 2016.

[133] A. Ghanizadeh, "Efficacy of N-acetylcysteine augmentation on obsessive compulsive disorder: a multicenter randomized double blind placebo controlled clinical trial," Iranian Journal of Psychiatry, vol. 12, no. 2, pp. 134-141, 2017.

[134] L. Smith, D. K. Tracy, and G. Giaroli, "What future role might $\mathrm{N}$-Acetyl-Cysteine have in the treatment of obsessive compulsive and grooming disorders?: a systematic review," Journal of Clinical Psychopharmacology, vol. 36, no. 1, pp. 57-62, 2016.

[135] D. L. C. Costa, J. B. Diniz, G. Requena et al., "Randomized, double-blind, placebo-controlled trial of $\mathrm{N}$-acetylcysteine augmentation for treatment-resistant obsessive-compulsive disorder," Journal of Clinical Psychiatry, vol. 78, no. 7, pp. e766-e773, 2017.

[136] M. Berk, D. L. Copolov, O. Dean et al., "N-acetyl cysteine for depressive symptoms in bipolar disorder--a double-blind 
randomized placebo-controlled trial," Biological Psychiatry, vol. 64, no. 6, pp. 468-475, 2008.

[137] J. E. Grant, B. L. Odlaug, and S. W. Kim, "N-acetylcysteine, a glutamate modulator, in the treatment of trichotillomania: a double-blind, placebo-controlled study," Archives of General Psychiatry, vol. 66, no. 7, pp. 756-763, 2009.

[138] M. Berk, C. S. Gama, G. S. Malhi et al., "Nail-biting stuff? The effect of N-acetyl cysteine on nail-biting," CNS Spectrums, vol. 14, no. 7, pp. 357-360, 2009.

[139] B. L. Odlaug and J. E. Grant, "N-acetyl cysteine in the treatment of grooming disorders," J Clin Psychopharmacol, vol. 27, no. 2, pp. 227-229, 2007.

[140] Y. M. Kupchik, K. Moussawi, X.-C. Tang et al., "The effect of $\mathrm{N}$-acetylcysteine in the nucleus accumbens on neurotransmission and relapse to cocaine," Biological Psychiatry, vol. 71, no. 11, pp. 978-986, 2012.

[141] R. L. Tomko, J. L. Jones, S. E. Back et al., "N-acetylcysteine: a potential treatment for substance use disorders," Current Opinion in Psychiatry, vol. 17, no. 6, pp. 30-36, 2018.

[142] S. Kasperczyk, "Effect of N-acetylcysteine administration on the expression and activities of antioxidant enzymes and the malondialdehyde level in the blood of lead-exposed workers," Environmental Toxicology and Pharmacology, vol. 37, no. 2, pp. 638-647, 2014.

[143] A. Giampreti, D. Lonati, B. Ragghianti et al., "N-AcetylCysteine as effective and safe chelating agent in metal-onmetal hip-implanted patients: two cases," Case Reports in Orthopedics, vol. 2016, Article ID 8682737, 7 pages, 2016.

[144] P. Hemalatha, A. G. Reddy, Y. R. Reddy, and P. Shivakumar, "Evaluation of protective effect of $\mathrm{N}$-acetyl cysteine on arsenic-induced hepatotoxicity," Journal of Natural Science, Biology and Medicine, vol. 4, no. 2, pp. 393-395, 2013.

[145] D. S. Martin, S. E. Willis, and D. M. Cline, "N-acetylcysteine in the treatment of human arsenic poisoning," Journal of the American Board of Family Medicine, vol. 3, no. 4, pp. 293296, 1990.

[146] K. Sooklert, S. Nilyai, R. Rojanathanes et al., "N-acetylcysteine reverses the decrease of DNA methylation status caused by engineered gold, silicon, and chitosan nanoparticles," International Journal of Nanomedicine, vol. 14, pp. 45734587, 2019.

[147] Š. Šalamon, B. Kramar, T. P. Marolt, B. Poljšak, and I. Milisav, "Medical and dietary uses of N-Acetylcysteine," Antioxidants (Basel), vol. 8, no. 5, 2019.

[148] T. T. Niemi, "The effect of N-acetylcysteine on blood coagulation and platelet function in patients undergoing open repair of abdominal aortic aneurysm," Blood Coagulation and Fibrinolysis, vol. 17, no. 1, pp. 29-34, 2006.

[149] D. Ardissino, S. Savonitto, P. A. Merlini et al., "Effect of transdermal nitroglycerin or $\mathrm{N}$-acetylcysteine, or both, in the long-term treatment of unstable Angina pectoris," Journal of the American College of Cardiology, vol. 29, no. 5, pp. 941947, 1997.

[150] S. C. De Rosa, "N-acetylcysteine replenishes glutathione in HIV infection," European Journal of Clinical Investigation, vol. 30, no. 10, pp. 915-929, 2000. 